\title{
Colossal Effects in Transition Metal Oxides can be caused by Intrinsic Inhomogeneities
}

\author{
J. Burgy, ${ }^{1}$ M. Mayr, ${ }^{1}$ V. Martin-Mayor, ${ }^{2}$ A. Moreo,${ }^{1}$ and E. Dagotto ${ }^{1}$ \\ ${ }^{1}$ National High Magnetic Field Lab, Florida State University, Tallahassee, FL 32306, USA \\ ${ }^{2}$ Dipartimento di Fisica, Università di Roma "La Sapienza", Piazzale Aldo Moro 2, 00185 Roma, Italy
}

(Dated: November 20, 2018)

\begin{abstract}
The influence of quenched disorder on the competition between ordered states separated by a first-order transition is investigated. A phase diagram with features resembling quantum-critical behavior is observed, even using classical models. The low-temperature paramagnetic regime consists of coexisting ordered clusters, with randomnly oriented order parameters. Extended to manganites, this state is argued to have a colossal magnetoresistance effect. A scale $\mathrm{T}^{*}$ for cluster formation is discussed. This is the analog of the Griffiths temperature, but for the case of two competing orders, producing a strong susceptibility to external fields. Cuprates may have similar features, compatible with the large proximity effect of the very underdoped regime.
\end{abstract}

PACS numbers: 71.10.-w, 75.10.-b, 75.30.Kz

Complex phenomena such as "colossal" magnetoresistance (CMR) in manganites and high temperature superconductivity (HTS) in cuprates have challenged our understanding of correlated electrons 值. Recent developments unveiled a previously mostly ignored aspect of doped transition-metal-oxides (TMO): these systems are intrinsically inhomogeneous, even in the best crystals. (i) The evidence in the CMR context is overwhelming. Experiments and theory provide a picture where competing ferromagnetic (FM) and charge-ordered (CO) states form microscopic and/or mesoscopic coexisting clusters [2, 3. Exciting recent experiments [4] identified features referred to as a "quantum critical point" (QCP) [5] - defined as the drastic reduction of ordering temperatures near the zero temperature $(\mathrm{T}=0)$ transition between ordered states - by modifying the A-site cation mean-radius $\left\langle r_{A}\right\rangle$ by chemical substitution at fixed hole density (left inset of Fig. 1). The paramagnetic state in the QCP region - where the Curie temperature $\mathrm{T}_{\mathrm{C}}$ is the lowest - is crucial to understand CMR phenomenology, producing the largest CMR ratio [1, 2, 3. 3. (ii) In the HTS context, scanning tunneling microscopy (STM) studies of superconducting (SC) Bi2212 revealed a complex surface with nm-size coexisting clusters [6]. Underdoped cuprates also appear to be inhomogeneous sal" proximity effect (CPE) was reported on underdoped $\mathrm{YBa}_{2} \mathrm{Cu}_{3} \mathrm{O}_{6+\mathrm{x}}$ over large distances [8].

In this paper, the competition between two ordered states in the presence of quenched disorder is investigated. These states are assumed sufficiently "different" that their low- $\mathrm{T}$ transition in the clean limit has firstorder characteristics. The approach has similarities with the classical work of Imry and Ma [9]. From the general considerations, doped TMOs are here considered, with intrinsic disorder caused by chemical substitution. For Mn-oxides, a possible rationalization of the CMR effect is discussed, with predictions including a scale $\mathrm{T}^{*}$ for cluster formation - the analog of the Griffiths tempera- ture 10 but in the regime of competing orders. For underdoped $\mathrm{Cu}$-oxides, a similar inhomogeneous picture is proposed. The calculations are mainly carried out using a two dimensional (2D) toy model of Ising spins, but similar data in three dimensions (3D) and for the one-orbital manganite model have also been gathered. Then, our conclusions appear valid for a variety of models with competing orders. The actual Hamiltonian employed here, defined on a square/cubic lattice (spacing $\mathrm{a}=1$ ) and with Ising variables, is $\mathrm{H}=\mathrm{J}_{1} \sum_{\langle\mathbf{i} \mathbf{j}\rangle} \mathrm{S}_{\mathbf{i}}^{\mathbf{z}} \mathrm{S}_{\mathbf{j}}^{\mathbf{z}}+\mathrm{J}_{2} \sum_{\langle\mathbf{i m}\rangle} \mathrm{S}_{\mathbf{i}}^{\mathrm{z}} \mathrm{S}_{\mathbf{m}}^{\mathrm{z}}$ $+\mathrm{J}_{4} \sum_{\langle\mathbf{i} \mathbf{n}\rangle} \mathrm{S}_{\mathbf{i}}^{\mathrm{z}} \mathrm{S}^{\mathrm{z}} \mathbf{n}$, in a standard notation. Sites $\langle\mathbf{i} \mathbf{j}\rangle$ are at distance 1 (usual nearest-neighbors), $\langle\mathbf{i m}\rangle$ at distance $\sqrt{2}$, and $\langle\mathbf{i n}\rangle$ at distance $\sqrt{5}$. The three couplings are antiferromagnetic $(\mathrm{AF})$. More than one coupling is needed to generate two competing $\mathrm{T}=0$ states, and $\mathrm{J}_{1}$ and $\mathrm{J}_{2}$ are the natural ones. However, the clean-limit first-order transition between those states was found to be more robust if a small $\mathrm{J}_{4} \sim 0.2 \mathrm{~J}_{1}$ coupling is added. The resulting competing states $\mathrm{O}_{1}$ and $\mathrm{O}_{2}$ are an AF state for low $\mathrm{J}_{2} / \mathrm{J}_{1}$, and a "collinear" AF state with rows (or columns) of spins up and down for large $J_{2} / J_{1}$ [11]. The main features of the toy model phase diagram are common to a variety of models with competing tendencies.

The toy model phase diagram, without disorder, is shown in Fig. 1, and it has the expected shape: the ordering temperatures decrease and meet at the clean-limit critical coupling $\mathrm{J}_{2 \mathrm{c}}=0.7 \mathrm{~J}_{1}$, and the low- $T$ transition was found to be clearly first-order. The most interesting result in Fig. 1 is the influence of disorder on the clean-limit diagram. The first-order transitions become continuous with sufficiently large disorder, in agreement with previous work [12]. Critical temperatures far from $\mathrm{J}_{2 \mathrm{c}}$ are not affected much by the disorder strengths considered. However, a drastic reduction is observed near $\mathrm{J}_{2 \mathrm{c}}$. In fact, the Monte Carlo (MC) results suggest that the obtained phase diagram is similar to the insets Fig. 1 for Mn- and $\mathrm{Cu}$-oxides. With increasing disorder strength $\mathrm{W}$, either a first-order line separating the competing phases still sur- 


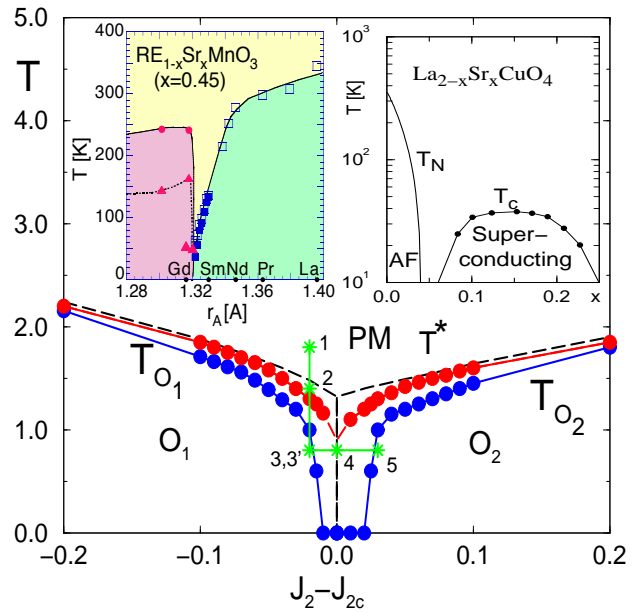

FIG. 1: Phase diagram of the $2 \mathrm{D} \mathrm{J}_{1}-\mathrm{J}_{2}-\mathrm{J}_{4}$ toy model used to analyze the competition between ordered states. Results on $32^{3}$ lattices (not shown) lead to a qualitatively similar phase diagram. $\mathrm{J}_{1}=1$ is the scale, and $\mathrm{J}_{4}$ is fixed to 0.2 . Disorder is incorporated such that $\mathrm{J}_{2}(\mathbf{i j})$ at a link joining sites $\mathbf{i}$ and $\mathbf{j}$ is uniformly distributed between $\mathrm{J}_{2}-\mathrm{W} / 2$ and $\mathrm{J}_{2}+\mathrm{W} / 2$. Blue (red) curve corresponds to $\mathrm{W}=1.5$ (0.75). Dashed black lines are the result without disorder $\mathrm{W}=0$, and $\mathrm{T}^{*}$ denotes the clean-limit transition. A Metropolis algorithm was used, on up to $512^{2}$ lattices, calculating (i) the largest ordered cluster size [J. Hoshen et al., Phys. Rev. B14, 3438 (1976)] and (ii) the order parameters for AF and collinear phases with a spin structure factor maximized at momenta $(\pi, \pi)$ and $(\pi, 0)$ $(0, \pi)$, respectively. Ten or more realizations of disorder were used, found to be sufficient for large systems. Insets are the phase diagrams of Mn-oxides in the FM-CO competition region [4], and of the single-layer $\mathrm{Cu}$-oxide in standard notation [J. B. Torrance et al., Phys. Rev. B40, 8872 (1989)]. Points 1-5 are explained in Fig. 2.

vives at $\mathrm{J}_{2 \mathrm{c}}$ (red points), as in manganites, or a disordered region of finite $\mathrm{J}_{2}$ width opens at $\mathrm{T}=0$ (blue points), as in single-layer cuprates. Note that the ordering temperatures exactly meet at $\mathrm{T}=0$ for only one fine-tuned $\mathrm{W}$. However, the overall shape of the phase diagram resembles QCP behavior in a robust range of W. For this reason, our results are qualitatively described as inducing "quantum-critical-point like" behavior, not a rigorous expression but hopefully descriptive enough to be useful.

Sufficiently strong quenched disorder will smooth firstorder transitions [12]. At $\mathrm{J}_{2 \mathrm{c}}$, this should occur at infinitesimal $\mathrm{W}$ in $2 \mathrm{D}$ [9], while a finite $\mathrm{W}$ is needed in $3 \mathrm{D}$. Then, at $\mathrm{J}_{2 \mathrm{c}}$ and with finite temperature, a paramagnetic state must be generated with growing disorder. Note also that our toy model is classical, but QCP-like behavior is nevertheless generated [13].

Figure 1 is the result of a systematic computational effort. As example, in Fig. 2a-b, the AF order parameter vs $\mathrm{T}$ is shown for $\mathrm{J}_{2}$ values outside and inside the coupling range where a $\mathrm{T}=0$ disordered regime is obtained. For $\mathrm{J}_{2}=0.69$ note the order-parameter cancel-

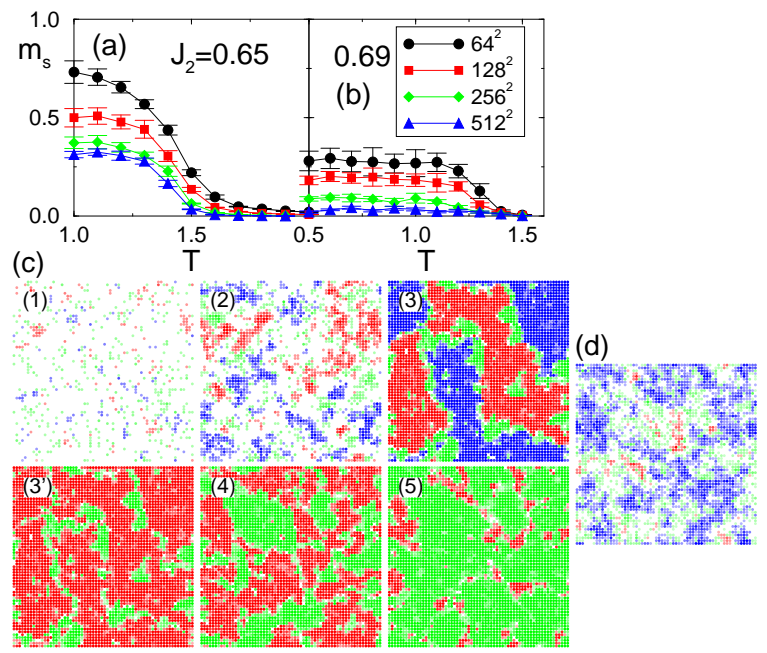

FIG. 2: AF order parameter vs $\mathrm{T}$ for the toy model at fixed $\mathrm{W}=1.5$, using several lattice sizes with periodic boundary conditions. (a) corresponds to $\mathrm{J}_{2}=0.65$ and (b) to $\mathrm{J}_{2}=0.69$. Note in the latter the order parameter rapid suppression as the size grows. (c) Typical spin configurations representative of dominant 2D states. Shown are averages over 10 measurements, in about $100 \mathrm{MC}$ sweeps to avoid correlations, after thermalizing with thousands of sweeps. Very similar results were obtained in $3 \mathrm{D}$ simulations. $(\mathbf{1}, \mathbf{2}, \mathbf{3})$ are at $\mathrm{J}_{2}=0.68$, and $\mathrm{T}=2.00,1.45$ (near the resistance peak, see Fig. 3), and 0.80, respectively (see Fig. 1). Green regions have collinear order, while red and blue indicate Néel and "anti-Néel" order. The last two differ in the staggered order parameter sign, i.e. they intuitively are $\uparrow \downarrow \uparrow \downarrow \ldots$ and $\downarrow \uparrow \downarrow \uparrow \ldots$. The white does not have a dominant order after the MC sweeps considered here. Green/red/blue pale regions have weak order. $\left(\mathbf{3}^{\prime}, \mathbf{4}, \mathbf{5}\right)$ corresponds to $\mathrm{T}=0.8$ and $\mathrm{J}_{2}=0.68,0.70$ and 0.73 , respectively (see Fig. 1 ), and the Néel and anti-Néel states are here given the same color (red), while green remains collinear. (d) Typical spin configuration at staggered field $\mathrm{H}_{\mathrm{s}}=0.01, \mathrm{~J}_{2}=0.68, \mathrm{~W}=1.5$, and $\mathrm{T}=1.45$.

lation with increasing size (this coupling is not critical at $\mathrm{T} \neq 0$ ). Representative spin configurations are in Fig. 2c. Keeping $\mathrm{J}_{2}$ constant and varying $\mathrm{T}$, three regimes are observed: (1) A high-T regime, where the system is disordered after MC time averaging. (2) An intermediate region $\mathrm{T}_{\mathrm{O}_{1}}<\mathrm{T}<\mathrm{T}^{*}$ with preformed clusters, but with uncorrelated order parameters giving a globally paramagnetic state, similar to the Griffiths phase. (3) A low-T regime where the clusters from (2) grow in size, although the disorder is uncorrelated from link to link, and percolate upon cooling. Note that clusters with different signs for the order parameter are separated by thin regions of the competing phase, providing a possible mechanism for stabilizing domain walls. Considering now fixed low- T but changing $\mathrm{J}_{2}$, configurations $\left(3^{\prime}, 4,5\right)$ are obtained. In this case, just the two ordered phases are in competition (no white regions), and the transition between phases appears percolative-like.

The main features of the results Figs. 1-2 - shape, clustered structure, the QCP-like behavior - are believed 


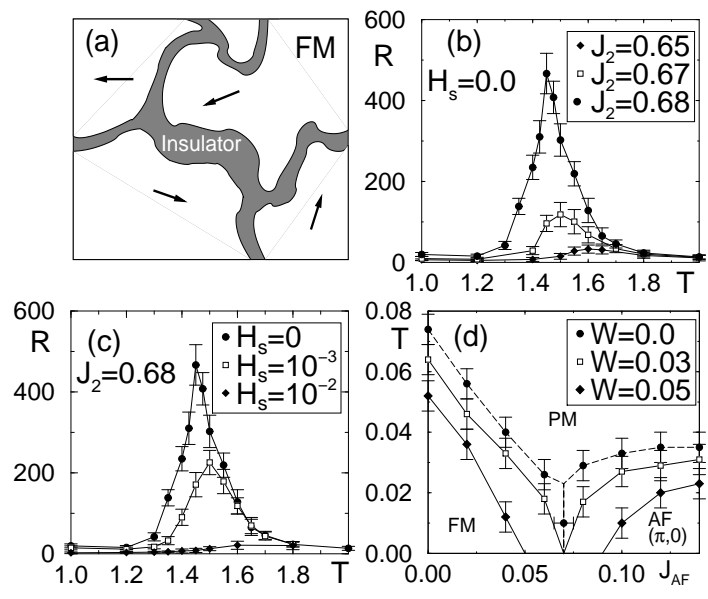

FIG. 3: (a) Proposed state for Mn-oxides in the CMR regime. (b) Resistance of the toy model after the equivalence to manganite states is used (see text), using a $256^{2}$ lattice, $\mathrm{W}=1.5$, at the couplings indicated. The calculation is carried out by transforming a spin configuration into a resistor network, with nodes centered at plaquettes (\# nodes $=1 / 4$ \# sites) and resistors between them. The values of the conductances of these resistors were established using $B B=R R=1.0, B R=0$, $\mathrm{WW}=0.3, \mathrm{GG}=\mathrm{BG}=\mathrm{RG}=\mathrm{WG}=0, \mathrm{WB}=\mathrm{WR}=0.5$, where $\mathrm{B}$, $\mathrm{R}, \mathrm{G}$, and $\mathrm{W}$ stand for blue, red, green and white regions (see Fig. 2c), $\mathrm{AA}^{\prime}=\alpha$ means that the resistor between plaquettes in the $\mathrm{A}(=\mathrm{B}, \mathrm{R}, \mathrm{G}, \mathrm{W})$ and $\mathrm{A}^{\prime}$ states has value $\alpha$ (arbitrary units), and $\mathrm{AA}^{\prime}=\mathrm{A}^{\prime} \mathrm{A}$. Other values for $\mathrm{WW}$ and $\mathrm{WB}$ lead to similar results, and $\mathrm{BB}$ defines the scale. Note that the conductivity should be spin dependent, and a BR link (when an electron moves from a spin-up to a spin-down region) has zero conductance. The algorithm used to obtain the total conductance is exact 14]. (c) Resistance (arbitrary units) vs T, at external fields $\mathrm{H}_{\mathrm{s}}$ indicated, using a $256^{2}$ lattice, $\mathrm{J}_{2}=0.68$, and $\mathrm{W}=1.5$. (d) $\mathrm{T}_{\mathrm{C}}^{*}$ results for the one-orbital manganite model using $8^{2}$ and $16^{2}$ clusters, density $x=0.5$, infinite Hund coupling, and hopping $\mathrm{t}=1$. With disorder, the $\mathrm{J}_{\mathrm{AF}}$ couplings are randomly distributed between $\mathrm{J}_{\mathrm{AF}}-\mathrm{W}$ and $\mathrm{J}_{\mathrm{AF}}+\mathrm{W}$ ( $\mathrm{W}$ indicated). In practice, $\mathrm{T}_{\mathrm{C}}^{*}$ was defined when the spin correlations at distance $\sqrt{2}$ dropped below $40 \%$ of the maximum value.

to be qualitatively general. In fact, simulations of oneorbital models (below) and other models studied in this effort give a similar phase diagram. Of course, the analogy should not be taken too far, e.g. critical exponents may not be universal since the Ising model underlying symmetries are quite different from those of realistic systems. However, it is worth investigating the consequences of the general phase diagram Fig. 1 for materials where two states strongly compete, such as in Mn-oxides. In this context, if a simulation of a realistic model with FM and $\mathrm{AF}$ phases on a huge lattice were possible, FM and AF clusters analogous of Fig. 2 would be found. Then, a reasonable way to bypass that (currently impossible) computational effort is to simply translate Fig. 2 into manganite language. This is a speculation, but hopefully the essence of the problem is preserved by the procedure. The proposed translation links order $\mathrm{O}_{1}$ with ferromag- netism, with order parameters pointing in different directions for different clusters, while $\mathrm{O}_{2}$ corresponds to charge-ordering. Translating Fig. 2c(2) into Mn-oxide language leads schematically to Fig. 3a, our proposed CMR state. The preformed FM clusters have uncorrelated moment orientations, and zero global magnetization. Note also that the "depth" of the QCP-like feature is not universal, it depends on the disorder strength.

To test the relevance of Fig. 3a to CMR manganites, a resistor network calculation was set up. Translating to Mn-oxide language, as explained before, the MC generated configurations were mapped into a resistance grid (see caption of Fig. 3b). For up (down) spins, the blue regions of Fig. $2 \mathrm{c}$ - analog of positive magnetization FM clusters - have high (low) conductivity, the red regions have low (high) conductivity, and the green regions are insulating. The Kirchoff equations were solved exactly, leading to the results Fig. 3b. In agreement with intuition, the non-percolated state Fig. 2c(2) has a large resistance for both spins up and down, while the percolated low- $\mathrm{T}$ or disordered high- $\mathrm{T}$ states have far better conductance. Note that the resistance peak intensity increases as the ordering temperature is reduced varying $\mathrm{J}_{2}$, analog of $\left\langle r_{A}\right\rangle$, closer to the QCP-like regime.

The rotation of the large moments of the preformed FM clusters (Fig. 3a) may occur with small magnetic fields. These effects are mimicked in the toy model using a staggered external field $\mathrm{H}_{\mathrm{s}}$ which favors $\mathrm{O}_{1}$ clusters with order parameter $\mathrm{M}_{\mathrm{s}}>0$ (blue, Fig. 2c) to the detriment of $\mathrm{O}_{1}$ clusters with $-\mathrm{M}_{\mathrm{s}}$ (red, Fig. 2c), or $\mathrm{O}_{2}$ regions. Figure $2 \mathrm{~d}$ confirms the rapid generation of positive $\mathrm{O}_{1}$ order in the region $\mathrm{T}_{\mathrm{O}_{1}}<\mathrm{T}<\mathrm{T}^{*}$ with tiny fields $0.01 \mathrm{~J}_{1}$. This severely affects transport (Fig. 3c), i.e. a modest field transforms the intermediate $\mathrm{T}$ cluster state into a fairly uniform state with robust conductance. Results Fig. 3cthe main results of this paper - are similar to those found in Mn-oxides, and a huge MR ratio $\left[\mathrm{R}(0)-\mathrm{R}\left(\mathrm{H}_{\mathrm{s}}\right)\right] / \mathrm{R}\left(\mathrm{H}_{\mathrm{s}}\right)$ of $\sim 4 \times 10^{3} \%$ was obtained at $\mathrm{H}_{\mathrm{s}}=10^{-2}$ 15.

In addition, there are already experimental indications in Mn-oxides for the existence of a temperature scale $\mathrm{T}^{*}$ for uncorrelated cluster formation [16], which should be ubiquitous in low-bandwidth manganites [17.

The phase diagram Fig. 1 is representative of more realistic models. Fig. 3d contains MC results for the oneorbital model [18], in which the coupling $\mathrm{J}_{\mathrm{AF}}$ between localized spins is varied to induce a metal-insulator transition [3]. Without disorder, the $\mathrm{T} \sim 0$ transition is known to be first-order between FM and AF states, the latter with rows or columns of spins up and down [3]. The "characteristic" ordering temperatures $\mathrm{T}_{\mathrm{C}}^{*}$, at which spin correlations become robust upon cooling, are shown vs. $\mathrm{J}_{\mathrm{AF}}$. Note the similarity with Fig. 1.

The results in Figs. 1-2 can also be adapted to cuprates. The high-Tc phase diagram (inset Fig. 1) shows a suppression of AF and $\mathrm{SC}$ order in a region usually labeled "spin-glass", whose origin is unclear. Consid- 

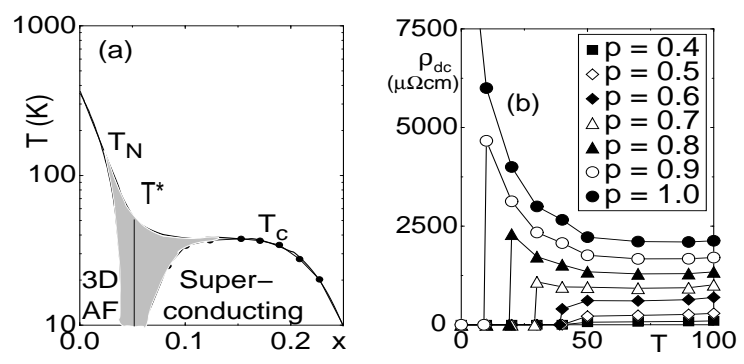

FIG. 4: (a) Conjectured HTS phase diagram. Black lines should be the actual phase boundaries without disorder. The shaded region is conjectured to have metallic (SC) and insulating (AF) coexisting regions in the real materials. (b) Resistivity $\rho_{a b}$ vs T, from a random-resistor network calculation as in Ref. 22. where details can be found. A $50 \times 50$ cluster was used, with $\rho_{a b}$ for insulating (optimal doping) fraction $\mathrm{p}=1.0(0.0)$ taken from LSCO $\mathrm{x}=0.04(0.15)$ data [H. Takagi et al , Phys. Rev. Lett. 09, 2975 (1992). See also Y. Ando et al., cond-nato104169. The inset labets are the p fractions at $100 \mathrm{~K}$, all of which are smoothly reduced with decreasing $\mathrm{T}$ until percolation to a SC state occurs at $\mathrm{p}=0.5$.

ering these diagrams together with the CPE results [8], it is conjectured that the very underdoped cuprate state may not be homogeneous but results from a SC vs. doped AF competition after disorder is considered. Inhomogeneities (clusters) should be present even within ordered phases [Fig. 2c(3)]. Stripe states are candidates for the doped AF state 19].

The proposed clean-limit phase diagram is in Fig. 4a, with a vertical first-order transition line, as cuprates have upon electron doping [20], and heavy fermions with varying pressure. The shaded region may contain a mixture of stripe-like and preformed SC islands [21]. Due to the general character of the discussion of Figs. 1-2, colossal effects should be ubiquitous when ordered phases compete, and they could appear in cuprates as well. CPE [8] could be a manifestation, with preformed SC clusters percolating under the influence of nearby SC materials. To further check this hypothesis, Fig. 4 d contains results of a phenomenological random-resistor calculation of resistivity vs $\mathrm{T}$ [22] in rough agreement with experiments [23].

Summarizing, the results presented in this paper [24] suggest that "colossal" effects in TMO's could originate in intrinsic inhomogeneities. These large effects may be more general than previously anticipated. In our studies, the analog of the classical Griffiths regime - usually associated with weak effects - is here much more robust, strongly susceptible to external fields.

This work was supported by NSF-DMR-9814350 and the Computational Science and Information Technology school at Florida State University. The authors thank Y.
Ando, D. N. Argyriou, S. L. Cooper, J. C. S. Davis, R. Decca, A. Feiguin, D. Gingold, N. Nagaosa, S. Sachdev, T. Senthil, P. Schiffer, and Y. Tokura for comments.

[1] Y. Tokura and N. Nagaosa, Science 288, 462 (2000).

[2] J. Lynn et al., Phys. Rev. Lett. 76, 4046 (1996); J. De Teresa et al., Nature 386, 256 (1997); J. Fernandez-Baca et al., Phys. Rev. Lett. 80, 1316 (1998); M. Uehara et al., Nature 399, 560 (1999); M. Fäth et al., Science 285, 1540 (1999); I. Deac et al., Phys. Rev. B63, 172408 (2001).

[3] E. Dagotto et al., Phys. Reports 344, 1 (2001).

[4] Y. Tokura et al., talk APS March meeting, Seattle, 2001.

[5] See Quantum Phase Transitions, by S. Sachdev, Cambridge University Press, 1999.

[6] S. H. Pan et al. submitted to Nature; C. Howald et al., cond-mat/0101251; K.M. Lang et al., preprint.

[7] M.-H. Julien et al., Phys. Rev. B63, 144508 (2001); A. W. Hunt et al., cond-mat/0011380; Y. Sidis et al., condmat/0101095: P. M. Singer et al., cond-mat/0108291.

[8] R. S. Decca et al., Phys. Rev. Lett. 85, 3708 (2000).

[9] Y. Imry and S. Ma, Phys. Rev. Lett. 35, 1399 (1975); E. Shimshoni et al., Phys. Rev. Lett. 80, 3352 (1998).

[10] R. B. Griffiths, Phys. Rev. Lett. 23, 17 (1969).

[11] D. P. Landau, Phys. Rev. B21, 1285 (1980).

[12] M. Aizenman and J. Wehr, Phys. Rev. Lett. 62, 2503 (1989); K. Hui and A. N. Berker, Phys. Rev. Lett. 62, 2507 (1989). See also Y. Imry and M. Wortis, Phys. Rev. B19, 3580 (1979); J. Cardy, cond-mat/9806355.

[13] Quantum effects may be of relevance at low T [A. P. Young and H. Rieger, Phys. Rev. B53, 8486 (1996)].

[14] D. Frank and C. Lobb, Phys. Rev. B37, 302 (1988).

[15] Using a FM Ising model, P. Bastiaansen and H. Knops, J. Phys. Chem. Solids 59, 297 (1998) (BK) followed a procedure similar to ours to address CMR. The magnitude of the effect in this context is substantially smaller than in our calculations, where two phases are involved.

[16] Results compatible with a $\mathrm{T}^{*}$ well above $\mathrm{T}_{\mathrm{C}}$ exist in manganites [De Teresa et al. (Ref. (2]); D. N. Argyriou et al., Phys. Rev. B60, 6200 (1999)], and in EuO [C. S. Snow et al., cond-mat/0011527.

[17] Mixed-phase regimes have a pseudogap in the density of states [A. Moreo et al., Phys. Rev. Lett. 83, 2773 (1999)].

[18] J. L. Alonso et al., Nucl. Phys. B 596, 587 (2001).

[19] Frustrated phase separation without explicit quenched disorder could also further stabilize the effects described here [V. Emery and S. Kivelson, Physica C209, 597 (1993); J. Schmalian and P. Wolynes, Phys. Rev. Lett. 85, 836 (2000)].

[20] N. Harima et al., cond-mat/0103519.

[21] Preformed SC islands may have already been observed [I. Iguchi et al, Nature 412, 420 (2001)].

[22] M. Mayr et al., Phys. Rev. Lett. 86, 135 (2001).

[23] N. Ichikawa et al., Phys. Rev. Lett. 85, 1738 (2000). See also M. Gutmann et al., cond-mat/0009141; T. Egami, cond-mat/0102449. Fig. 4a is related to SO(5) ideas (S. C. Zhang, Science 275, 1089 (1997)).

[24] Details will be provided elsewhere. 